C2017, Elsevier. Licensed under the Creative Commons Attribution-NonCommercialNoDerivatives 4.0 International http://creativecommons.org/about/downloads

(c) (i) $\ominus$ 


\section{Graphical Abstract}

Greener synthesis using hydrogen peroxide

in ethyl acetate of alicyclic ring-fused

benzimidazoles and anti-tumour

benzimidazolequinones

Martin Sweeney, Michael Gurry, Lee-Ann J. Keane, Fawaz Aldabbagh*
Leave this area blank for abstract info.<smiles>[R]c1cc([R])c(N2CCCC2)c(N)c1[R]</smiles>

$R^{1}=H, E D G, E W G$
$\mathrm{H}_{2} \mathrm{O}_{2}$, EtOAc, reflux

Yields: $60-93 \%$

$n=1-4$

16 examples<smiles>[R2]O[C@@H](C)[C@@H](C)O[R]</smiles>

Many examples: no extractions \& no chromatography<smiles>O=C1C=CC(=O)c2c1nc1n2CC=[Te]1</smiles>

anti-tumour agent 



\title{
Greener synthesis using hydrogen peroxide in ethyl acetate of alicyclic ring-fused benzimidazoles and anti-tumour benzimidazolequinones
}

\author{
Martin Sweeney, Michael Gurry, Lee-Ann J. Keane, Fawaz Aldabbagh* \\ School of Chemistry, National University of Ireland, Galway, University Road, Galway, Ireland
}

ARTICLE INFO

Article history:

Received

Received in revised form

Accepted

Available online

Keywords:

Annulations

Green chemistry

Heterocycles

Quinones

\section{ABSTRACT}

\begin{abstract}
Environmentally-friendly and cost effective hydrogen peroxide in ethyl acetate was used to prepare in high yields pyrrolo[1,2- $a$ ] benzimidazoles from commercial $o$-(pyrrolidin-1-yl)anilines without the requirement for organic-aqueous extraction and chromatography. Six, seven and eight membered ring-fused analogues were similarly obtained in high yields with methanesulfonic acid required for the pyrido[1,2-a]benzimidazole. Anti-tumour benzimidazolequinone derivatives were obtained in high yield via the cyclization of 3,6dimethoxy-2-(cycloamino)anilines.
\end{abstract}

\section{Introduction}

The synthesis of privileged alicyclic ring-fused [1,2-a] benzimidazoles has attracted sustained attention, ${ }^{1-28}$ most notably because of their conversion to potent bioreductive anti-tumour benzimidazolequinones. $^{3,16,29}$ There are drawbacks to reported syntheses of alicyclic ring-fused benzimidazoles such as requirements for transition metals, ${ }^{1-9}$ prior synthesis of cyclization precursors, ${ }^{10-17}$ full equivalents of strong base, ${ }^{17-20}$ high molar mass hypervalent iodine reagents, ${ }^{21}$ and the formation of mixtures of isomeric products. ${ }^{22}$ The most convenient protocol remains the traditional oxidative cyclization of $o$-cyclic amine substituted anilines, which was first reported in the early 1960s using hydrogen peroxide in the presence of trifluoroacetic acid. ${ }^{23}$ Variations include converting o-tert-aminoacetanilide to benzimidazole using $\mathrm{H}_{2} \mathrm{O}_{2}$ in formic acid ${ }^{24,25}$ and ring-fused benzimidazole and imidazobenzimidazole were precipitated without the requirement for chromatography using Oxone in formic acid. ${ }^{26}$ Most recently, $\mathrm{H}_{2} \mathrm{O}_{2}$ in combination with hydrochloric or hydrobromic acid mediated the cyclization of $o$ cyclic amine substituted anilines to give respectively dichlorinated and dibrominated ring-fused benzimidazoles in high yields of $>80 \%$. $^{27}$

From a green chemistry and commercial perspective, there are significant advantages to using the low molar mass ubiquitous oxidant $\mathrm{H}_{2} \mathrm{O}_{2}$ in synthesis since the only effluent is water, and its cost is lower than conceivable alternatives. Hydrogen peroxide $(50 \% \mathrm{w} / \mathrm{v})$ in water has a $\mathrm{pH} \sim 4 .^{30}$

It was envisaged that the weak acidic property of $\mathrm{H}_{2} \mathrm{O}_{2}$ would negate the requirement for carrying out the cyclization in organic and mineral acids, as described in literature procedures. ${ }^{3,23-28}$ Recently the preparation of alicyclic ring-fused benzimidazoles in formic acid was reported at high temperatures using an iodine- mediated cyclization of $o$-nitro-t-anilines, where corrosive HI was generated in situ. ${ }^{28}$

Ethyl acetate is classified as a preferred solvent by Pfizer, ${ }^{31}$ while GSK give ethyl acetate high ratings for environmental impact, health and reactivity / stability. ${ }^{32}$ Herein is disclosed the oxidative cyclization of commercial $o$-(cycloamino)anilines to give five, six, seven and eight-membered alicyclic ring-fused [1,2- $a$ ]benzimidazoles using $\mathrm{H}_{2} \mathrm{O}_{2}$ in ethyl acetate. Our aim was as much as possible to make reactions compliant with green chemistry principles, ${ }^{33}$ including use of safer solvents and to minimize waste / effluent generation with most reactions requiring no aqueous extraction or chromatography. The $p$ dimethoxy substituted pyrrolo[1,2-a]benzimidazole and pyrido[1,2- $a$ ]benzimidazole were prepared in high yields from their respective anilines without the requirement for chromatography and transformed into the anti-tumour benzimidazolequinones. New large ring-fused analogues, azepino and azocino[1,2-a]benzimidazoles can be similarly prepared using $\mathrm{H}_{2} \mathrm{O}_{2}$ in ethyl acetate, and converted to the novel benzimidazolequinones.

\section{Results and discussion}

Optimized conditions used 20 equivalents of $\mathrm{H}_{2} \mathrm{O}_{2}$ in ethyl actetate facilitating the conversion of commercial 2-(pyrrolidin1-yl)anilines 1a-1i into 2,3-dihydro- $1 H$-pyrrolo[1,2a]benzimidazoles 2a-2i in isolated yields of $64-93 \%$ (Table 1). Longer reaction times were required for full conversion of anilines containing an electron-withdrawing substituent at the 5position. The yield of 2,3-dihydro- $1 H$-pyrrolo[1,2-

\footnotetext{
* Corresponding author. Tel.: +353 91 493120; fax: +353 91 525700;

E-mail address: Fawaz.Aldabbagh@nuigalway.ie (F. Aldabbagh)
} 
Table 1. Synthesis of 2,3-dihydro-1H-pyrrolo[1,2-a benzimidazoles $^{a}$

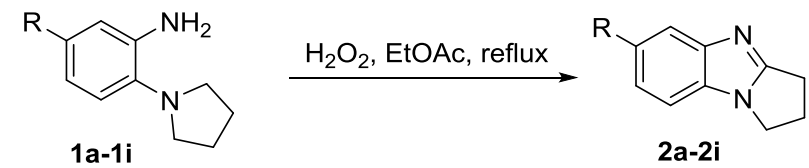

\begin{tabular}{clcc}
\hline aniline & $\mathbf{R}$ & time & yield (\%) \\
\hline 1a & $\mathrm{OMe}$ & $20 \mathrm{~min}$ & $\mathbf{2 a}, 79$ \\
$\mathbf{1 b}$ & $\mathrm{H}$ & $20 \mathrm{~min}$ & $\mathbf{2 b}, 80$ \\
$\mathbf{1 c}$ & $\mathrm{Me}$ & $40 \mathrm{~min}$ & $\mathbf{2 c}, 81$ \\
$\mathbf{1 d}$ & $\mathrm{NHAc}$ & $20 \mathrm{~min}$ & $\mathbf{2 d}, 64$ \\
$\mathbf{1 e}$ & $\mathrm{Br}$ & $2 \mathrm{~h}$ & $\mathbf{2 e}, 88$ \\
$\mathbf{1 f}$ & $\mathrm{F}$ & $50 \mathrm{~min}$ & $\mathbf{2 f}, 84$ \\
$\mathbf{1 g}$ & $\mathrm{CN}$ & $4 \mathrm{~h}$ & $\mathbf{2 g}, 85^{b}$ \\
$\mathbf{1 h}$ & $\mathrm{CF}_{3}$ & $8 \mathrm{~h}$ & $\mathbf{2 h}, 93$ \\
$\mathbf{1 i}$ & $\mathrm{NO}_{2}$ & $4 \mathrm{~h}$ & $\mathbf{2 i}, 71^{b}$ \\
\hline
\end{tabular}

${ }^{a}$ Reaction conditions: Aniline 1a-1i $(1.0 \mathrm{mmol}), \mathrm{H}_{2} \mathrm{O}_{2}(50 \% \mathrm{w} / \mathrm{v}, 20 \mathrm{mmol})$ and EtOAc $(5 \mathrm{~mL})$. When GC-MS indicated all the aniline was consumed, the reaction was quenched by addition of solid $\mathrm{Na}_{2} \mathrm{CO}_{3}(\sim 3 \mathrm{~g})$ and additional EtOAc $(20 \mathrm{~mL})$. The mixture was filtered and evaporated to give the ringfused $[1,2-a]$ benzimidazole. ${ }^{b}$ Included $\mathrm{MeSO}_{3} \mathrm{H}(0.5 \mathrm{mmol})$.

a]benzimidazole-6-carbonitrile (2g) was raised to $85 \%$ from $59 \%$, and the yield of 6-nitro-2,3-dihydro- $1 H$-pyrrolo[1,2a] benzimidazole (2i) was raised to $71 \%$ from $29 \%$ by addition of 0.5 equivalents of methanesulfonic acid (MSA). MSA undergoes biodegradation by forming $\mathrm{CO}_{2}$ and sulfate, and is considered a green acid due to its less toxic and corrosive nature. ${ }^{34}$ In all cases organic-aqueous extractions were avoided by addition of solid sodium carbonate to the reaction mixture. The quenched reaction was filtered with the ethyl acetate filtrate evaporated, and the solvent reused in further reactions. Our protocol circumvents the requirement for auxiliary solvents, allows solvent recycling and there is no aqueous effluent. The solid waste generated (containing sodium percarbonate) is sufficiently safe for disposal in landfill.

The formation of ring expanded analogues using the reaction conditions successfully applied to prepare pyrrolo[1,2a]benzimidazoles 2a-2i was investigated (Table 2). The sixmembered cyclization of 5-methoxy-2-(piperidiny-1-yl)aniline was slow without the addition of acid with an optimized yield of 75\% for 7-methoxy-1,2,3,4-tetrahydropyrido[1,2$a$ ]benzimidazole (3a) obtained after $1 \mathrm{~h}$ when one equivalent of MSA was added. Unfortunately the use of full equivalents of MSA necessitated the use of saturated sodium carbonate solution in an organic-aqueous extraction. Additional acid was not required for the seven and eight-membered cyclizations, which occurred in $65 \%$ and $73 \%$ yield respectively using $\mathrm{H}_{2} \mathrm{O}_{2}$ in ethyl acetate, however 3-methoxy-7,8,9,10-tetrahydro- $6 H$-azepino[1,2a]benzimidazole (4a) required purification using flash chromatography in order to remove unidentified impurities.

Table 2. Synthesis of five to eight-membered ring-fused [1,2a]benzimidazoles ${ }^{a}$

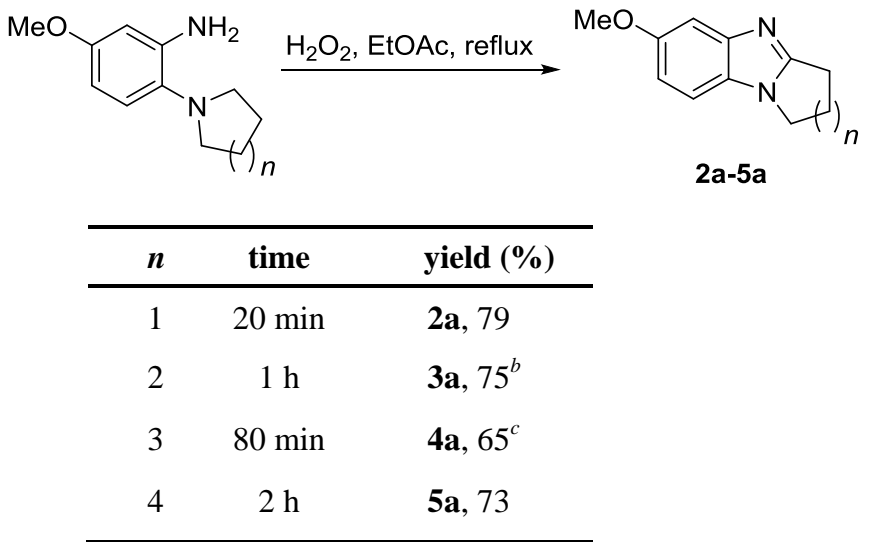

${ }^{a}$ Reaction conditions and work up is the same as Table $1 .{ }^{b}$ Included $\mathrm{MeSO}_{3} \mathrm{H}$ (1 mmol). Extraction with EtOAc $(20 \mathrm{~mL})$ and saturated $\mathrm{Na}_{2} \mathrm{CO}_{3}$ (aq.) was deemed necessary. ${ }^{c}$ Flash column chromatography required.

The conversion of 6,9-dimethoxy-1,2,3,4-tetrahydropyrido[1,2a]benzimidazole $\mathbf{8 b}$ into the six-membered ring-fused benzimidazolequinone $\mathbf{9 b}$ using hydrobromic acid-induced demethylation to give the hydroquinone in situ followed by room temperature oxidation with ferric chloride has been previously described by our group (Scheme 1). ${ }^{16}$ Pyrido[1,2a]benzimidazole $\mathbf{8 b}$ was however obtained using expensive and environmentally damaging $\mathrm{Bu}_{3} \mathrm{SnH}$-mediated radical cyclization of a benzeneselenide precursor. Furthermore, the scope of radical cyclizations is limited due to difficulties in forming constrained ring-fused systems such as pyrrolo[1,2-a]benzimidazole 8a.

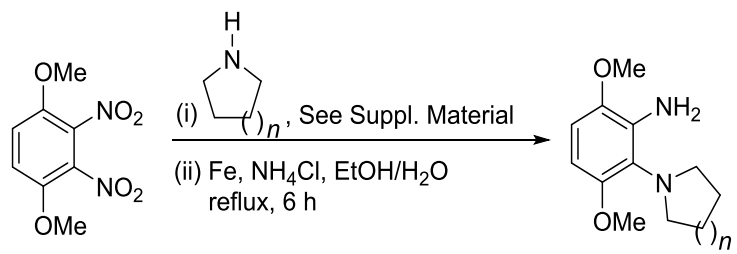

7a, $n=1(68 \%) 7 c, n=3(52 \%)$

$7 \mathrm{~b}, n=2(65 \%) 7 \mathrm{~d}, n=4(26 \%)$

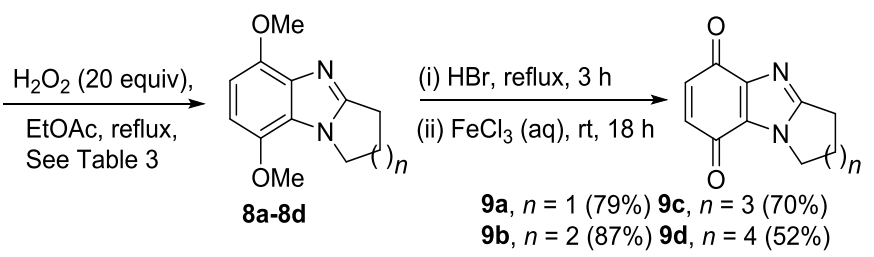

Scheme 1. Synthesis of five to eight-membered ring-fused benzimidazolequinones

A facile synthesis of anti-tumour agents 9a-9d is now disclosed starting from nucleophilic substitution of pyrrolidine, piperidine, azepane, and azocane onto 1,4-dimethoxy-2,3-dinitrobenzene followed by reduction to give the cyclization precursors $7 \mathbf{a}-7 \mathbf{d}$ in 26-68\% yield (over two synthetic steps in Scheme 1). In agreement with the above findings (Table 2), the five-membered cyclization proceeded efficiently, although slowly using $\mathrm{H}_{2} \mathrm{O}_{2}$ in EtOAc to give $8 \mathbf{a}$ in a yield of $81 \%$ over 18 h (Table 3 ). The analogous six-membered cyclization proved difficult using $\mathrm{H}_{2} \mathrm{O}_{2}$ alone. The addition of a full equivalent of MSA allowed the clean isolation of $\mathbf{8 b}$ in $80 \%$ yield after $12 \mathrm{~h}$ of reflux in ethyl acetate without the requirement for chromatography. It may be that the intermediate $N$-oxide of the six-membered ring tertiary amine is 
more stable through hydrogen bonding, ${ }^{26}$ requiring acidic conditions for oxidative cyclization. The seven and eight membered cyclizations of 3,6-dimethoxyanilines $\mathbf{7 c}$ and $\mathbf{7 d}$ proceeded in good yields $(60-72 \%)$ in $6 \mathrm{~h}$ in the presence of 1 equivalent of MSA with the absence of the acid leading to lower yields $(\sim 35 \%)$ and long reaction times as shown in Table 3. Benzimidazoles 8c and $\mathbf{8 d}$ were readily converted into novel potential anti-tumour agents azepino and azocino[1,2a] benzimidazolequinones $9 \mathbf{c}$ and $9 d$ in 52-70\% yield (Scheme 1), and will be the subject of future cytotoxicity evaluation by our group.

Table 3. Synthesis of five to eight-membered ring-fused dimethoxy substituted $[1,2-a]$ benzimidazoles $^{a}$

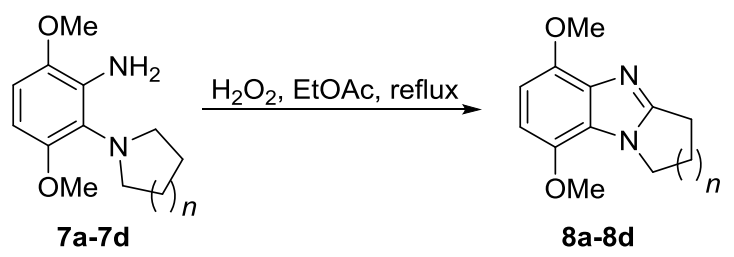

\begin{tabular}{cccl}
\hline aniline & time (h) & $\begin{array}{l}\mathbf{M e S O}_{\mathbf{3}} \mathbf{H} \\
(\mathbf{e q u i v})\end{array}$ & yield (\%) \\
\hline $\mathbf{7 a}$ & 18 & 0 & $\mathbf{8 a}, 81$ \\
$\mathbf{7 b}$ & 12 & 1 & $\mathbf{8 b}, 80^{b}$ \\
$\mathbf{7 c}$ & 24 & 0 & $\mathbf{8 c}, 34^{b, c}$ \\
$\mathbf{7 c}$ & 6 & 1 & $\mathbf{8 c}, 72^{b}$ \\
$\mathbf{7 d}$ & 36 & 0 & $\mathbf{8 d}, 35^{b, c}$ \\
$\mathbf{7 d}$ & 6 & 1 & $\mathbf{8 d}, 60^{b, c}$ \\
\hline
\end{tabular}

${ }^{a}$ Reaction conditions and work up is the same as Table 1. ${ }^{b}$ Extraction with EtOAc $(20 \mathrm{~mL})$ and saturated $\mathrm{Na}_{2} \mathrm{CO}_{3}$ (aq.) was deemed necessary. ${ }^{c}$ Flash column chromatography required

\section{Conclusion}

In summary nine pyrrolo[1,2-a]benzimidazoles have been prepared from commercial anilines using "acid-free" $\mathrm{H}_{2} \mathrm{O}_{2}$ in ethyl acetate protocol, where no aqueous-organic extraction or chromatography is required. Where reactions are slow environmentally acceptable MSA can be added. Six to eightmembered ring-fused benzimidazoles can be prepared using analogous conditions. The cyclization of 3,6-dimethoxy-2(cycloamino)anilines mostly required one equivalent of MSA in order to achieve high yields in a reasonable time. Nevertheless, the $\mathrm{H}_{2} \mathrm{O}_{2}$ in ethyl acetate protocol has considerable merit over reported routes to ring-fused benzimidazoles and benzimidazolequinones, which are more costly, environmentallydamaging, and often ineffective in preparing constrained and ring-expanded systems.

\section{Acknowledgments}

FA thanks the Irish Research Council (IRC) for a Government of Ireland Postgraduate Scholarship for Martin Sweeney and College of Science, National University of Ireland Galway (NUI Galway) for postgraduate scholarships for Michael Gurry and Lee-Ann J. Keane.

\section{Supplementary Material}

Supplementary data associated with this article can be found, in the online version, at $\mathrm{xxxxxx}$

\section{References}

1. Suschitzky H, Sutton ME, Tetrahedron 1968; 24: 4581-4587.

2. Anastasiou D, Campi EM, Chaouk H, Jackson WR. Tetrahedron 1992; 48: 7467-7478.

3. Zhou R, Skibo EB. J Med Chem. 1996; 39: 4321-4331.

4. Tan KL, Vasudevan A, Bergman RG, Ellman JA, Souers A J Org Lett. 2003; 5: 2131-2134.

5. Deng X, McAllister H, Mani NS. J Org Chem. 2009; 74: 57425745.

6. Alonso J, Halland N, Nazaré M, R'kyek O, Urmann M Lindenschmidt A. Eur J Org Chem. 2011; 234-237.

7. Liubchak K, Nazarenko K, Tolmachev A. Tetrahedron 2012; 68: 2993-3000.

8. Joardar S, Bhattacharyya A, Das S. Synthesis 2014; 46: 3121 3132.

9. $\quad$ Sun X, Lv X-H, Ye L-M, Hu Y, Chen Y-Y, Zhang X-J, Yan M. Org Biomol Chem. 2015; 13: 7381-7383.

10. Freedman AR, Payne DS, Day AR. J Heterocycl Chem. 1966; 3 : 257-259.

11. Haque MR, Rasmussen M. Tetrahedron 1997; 53: 6937-6958.

12. Aldabbagh F, Bowman WR. Tetrahedron 1999; 55: 4109-4122.

13. Allin SM, Bowman WR, Karim R, Rahman SS. Tetrahedron 2006; 62: 4306-4316.

14. Bastug G, Eviolitte C, Markó I E. Org Lett. 2012; 14: 3502-3505.

15. Coyle R, Fahey K, Aldabbagh F. Org Biomol Chem. 2013; 11: 1672-1682.

16. Lynch M, Hehir S, Kavanagh P, Leech D, O'Shaughnessy J, Carty MP, Aldabbagh F. Chem Eur J. 2007; 13: 3218-3226.

17. Nazarenko EG, Shyrokaya TI, Shvidenko EV, Tolmachev AA, Synth Commun. 2003; 33: 4303-4311.

18. Caroon JM, Fisher LE. Heterocycles 1991; 32: 459-467.

19. McClure JR, Custer JH, Schwarz HD, Lill DA. Synlett 2000; 5 : 710-712.

20. Baars H, Beyer A, Kohlhepp SV, Bolm C. Org Lett. 2013; 16: 536-539.

21. Huang J, He Y, Wang Y, Zhu Q. Chem Eur J. 2012; 18: 1396413967.

22. Chen J, Qu J, Zhang Y, Chen Y, Liu N, Chen B. Tetrahedron 2013; 69: 316-319.

23. Nair MD, Adams R. J Am Chem. 1961; 83: 3518-3521.

24. Meth-Cohn O, Smalley RK, Suschitzky H. J Chem Soc. 1963; 1666-1669.

25. Fahey K, Aldabbagh F. Tetrahedron Lett. 2008; 49: 5235-5237.

26. Fagan V, Bonham S, McArdle P, Carty MP, Aldabbagh F. Eur J Org Chem. 2012; 1967-1975.

27. Gurry M, Sweeney M, McArdle P, Aldabbagh F. Org Lett. 2015; 17: 2856-2859.

28. Nguyen TB, Ermolenko L, Al-Mourabit A. Green Chem. 2016; 18: 2966-2970.

29. Fahey K, O'Donovan L, Carr M, Carty MP, Aldabbagh F. Eur J Med Chem. 2010; 45: 1873-1879.

30. Eul W, Moeller A, Steiner N. Hydrogen Peroxide, Kirk-Othmer Encyclopedia of Chemical Technology, John Wiley \& Sons, Inc., 2001, p 43.

31. Alfonsi K, Colberg J, Dunn PJ, Fevig T, Jennings S, Johnson TA Kleine HP, Knight C, Nagy MA, Perry DA, Stefaniak M. Green Chem. 2008; 10: 31-36.

32. Henderson RK, Jiménez-González C, Constable DJC, Alston SR, Inglis GGA, Fisher G, Sherwood J, Binks SP, Curzons AD. Green Chem. 2011; 13: 854-862.

33. Anastas PT, Warner JC. Green Chemistry: Theory and Practice, Oxford University Press: New York, 1998, p 30.

34. Gernon MD, Wu M, Buszta T, Janney P. Green Chem. 1999; 1: 127-140. 